\title{
ASSOCIATION OF CAROTENOID INTAKE WITH PULMONARY FUNCTION
}

\author{
A Thesis \\ by \\ LAUREN YOURIM JUN
}

Submitted to the School of Graduate Studies

at Appalachian State University

in partial fulfillment of the requirements for the degree of

MASTER OF SCIENCE

May 2020

Department of Nutrition and Health Care Management 
A Thesis

by

LAUREN YOURIM JUN

May 2020

\section{APPROVED BY:}

Martin Root, $\mathrm{PhD}$

Chairperson, Thesis Committee

Jamie Griffin, $\mathrm{PhD}$

Member, Thesis Committee

Kyle Thompson, DCN, RDN

Member, Thesis Committee

Margaret Barth, $\mathrm{PhD}, \mathrm{MPH}$

Chairperson, Department of Nutrition and Health Care Management

Mike McKenzie, Ph.D.

Dean, Cratis D. Williams School of Graduate Studies 
Copyright by Lauren Jun 2020

All Rights Reserved 


\begin{abstract}
ASSOCIATION OF CAROTENOID INTAKE WITH PULMONARY FUNCTION Lauren Jun

B.S., Appalachian State University

Chairperson: Martin Root, Ph.D.
\end{abstract}

Lung function gradually declines as adults age, which can make breathing more difficult. Previous studies have reported enhanced lung function among older adults with high intake of carotenoid-rich foods. The aim of this study is to investigate the association between carotenoid-rich diet and lung function.

Data were taken from the Atherosclerosis Risk in Communities (ARIC) study, which included approximately 15,000 individuals aged 45-64 years at baseline from each of four counties in the United States. Food frequency questionnaire (FFQ) data were used to assess dietary intake of carotenoid-rich foods. Total carotenoids intake was calculated by adding five specific carotenoids - $\alpha$-carotene, $\beta$-carotene, $\beta$-cryptoxanthin, lycopene, and lutein/zeaxanthin. Pulmonary function was evaluated as the ratio of forced expiratory volume in one second $\left[\mathrm{FEV}_{1}\right]$ and forced vital capacity $[\mathrm{FVC}]$. Linear regression analysis was used to assess the association between intake of carotenoids and pulmonary function.

The association between the total carotenoid intake with pulmonary function was marginally positively significant; however, $\alpha$-carotene, $\beta$-carotene, and $\beta$-cryptoxanthin intakes were individually significantly associated with a higher $\mathrm{FEV}_{1} / \mathrm{FVC}$ ratio in study participants.

Dietary sources of pro-vitamin A carotenoids include yellow/orange fruits and vegetables such as oranges and sweet potatoes. Higher intake of these foods may improve pulmonary function. 


\section{Acknowledgments}

I would like to thank my committee chair, Dr. Martin Root, for his guidance and support throughout this process. I also appreciate Dr. Jamie Griffin and Dr. Kyle Thompson for serving on my committee. I would like to thank the Department of Nutrition and Health Care Management for providing an opportunity to facilitate this research. 


\section{Table of Contents}

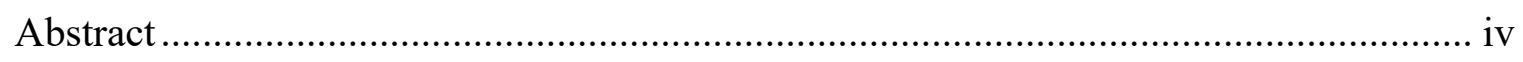

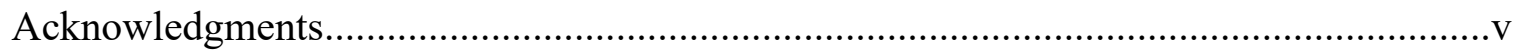

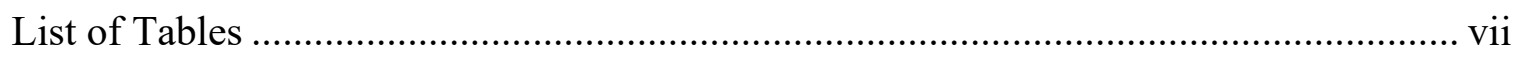

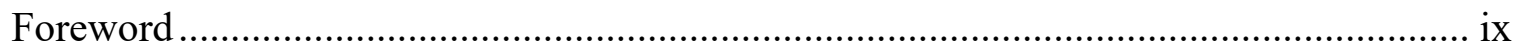

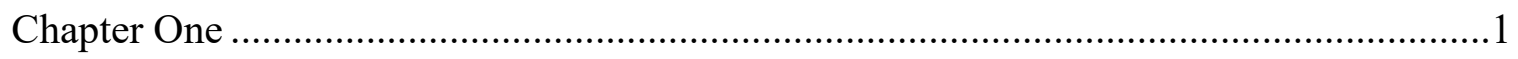

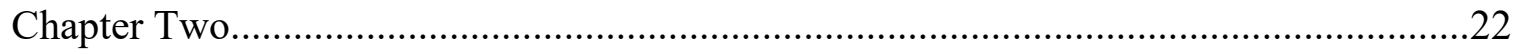

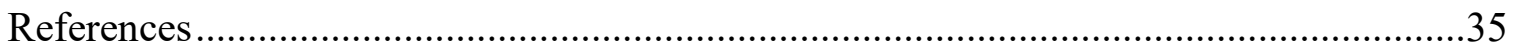

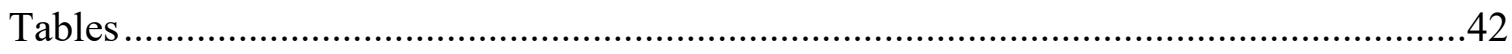

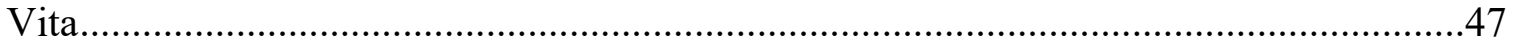




\section{List of Tables}

Table 1. Characteristics of Participants from the ARIC Cohort .....................................42

Table 2. Association Between Total Carotenoid Intake and $\mathrm{FEV}_{1} / \mathrm{FVC}$ Ratio .................43

Table 3. Association Between Individual Carotenoid Intake and $\mathrm{FEV}_{1} / \mathrm{FVC}$ Ratio .........44

Table 4. Servings of Carotene-Rich Fruits and Vegetables Across Smoking Status ....45

Table 5. Association Between Carotenoid-Rich Food Sources and FEV $/$ FVC Ratio .....46 


\section{Foreword}

Chapter 2 of this thesis will be submitted to Journal of the American College of

Nutrition, an international peer-reviewed journal owned by Taylor and Francis Group and

published by Taylor and Francis; it has been formatted according to the style guide for that journal. 


\section{Chapter One}

Chronic obstructive pulmonary disease (COPD) is the fourth leading cause of death in the world and is projected to be the third leading cause of death by 2020 (1). According to the Global Initiative for Chronic Obstructive Lung Disease, more than three million people died of COPD in 2012, which is six percent of all deaths globally (1). Based on large scale epidemiological studies, the number of COPD cases was estimated to be 384 million in 2010 , with a global prevalence of $11.7 \%(1)$.

COPD is an inflammatory disease leading to obstructive lung airways and airflow limitations (2) that interferes with normal breathing (3). These problems, caused by a narrowing airway lumen diameters (e.g., obstructive bronchiolitis) and parenchymal destruction (emphysema) develop as a result of varying perturbations in both airway and interstitial lung tissue (4). The chronic inflammation experienced in COPD causes structural changes, narrowing of the small airways and destruction of the lung parenchyma that leads to the loss of alveolar attachments to the small airways and decreases lung elastic recoil, diminishing the ability of the airways to remain open during expiration (1).

A COPD diagnosis is considered in any patient with the following symptoms of a chronic cough, sputum production, dyspnea, and a history of exposure to risk factors for the disease such as chemical or environmental exposure and family history. Diagnosis is confirmed by a spirometry test, which measures how fast air can move in and out of the lungs and how deeply a person can breathe (5). More specifically, the ratio of the forced expiratory volume in 1 second $\left(\mathrm{FEV}_{1}\right)$ to the total vital capacity $(\mathrm{FVC})$ is used to define the severity of the disease (6). COPD is partially classified by a $\mathrm{FEV}_{1} / \mathrm{FVC}$ ratio of less than 0.70 and an $\mathrm{FEV}_{1}$ less than $80 \%$ of that predicted by age, sex, ethnicity, and standing height (7). 
COPD is not common before the age of 40 , but symptoms of hypersecretion occur with increasing frequency after that age (4). In fact, more than $10 \%$ of individuals over 50 years of age develop COPD (4). At an older age, increased wall thickening, intraluminal mucus accumulation, smooth muscle hypertrophy, and small airway lining fluid changes may occur (4) leading to reduced lung function (4). Due to variable signs and symptoms of COPD and other similar conditions that are attributed to older age, irregularities in pulmonary function are often misdiagnosed or diagnosed too late (4).

COPD development is due mainly to biological factors such as inflammation and oxidative stress caused by free radicals, and inadequate intake of antioxidant-rich foods (8). Augmented pollution from rapid industrialization and increases in the smoking rates worldwide also increase risk for developing COPD (9). According to Scanlon et al. (10), risk factors to the development of COPD include tobacco smoking, poor initial lung function, advanced age, male sex, childhood respiratory illness, occupational respiratory exposures, air pollution, low educational attainment or socioeconomic status, and other familial factors. Individuals with COPD and smokers without airflow obstruction show an increased number of inflammatory cells infiltrating the adventitia of pulmonary muscular arteries.

Another epidemiological study investigated the prevalence and incidence of COPD by age, sex, and smoking status with 25 years of follow-up. COPD was diagnosed based on obstructive pre-bronchodilator spirometry $\left(\mathrm{FEV}_{1} / \mathrm{FVC}\right)$ according to the Global Initiative for Chronic Obstructive Lung Disease (GOLD) guidelines ( $\mathrm{FEV}_{1} / \mathrm{FVC}<70 \%$ ). The study found an increased overall incidence rate of COPD with age, higher in men than in women, and with smokers compared to non-smokers (11). 
In recent studies, cigarette smoke products have been shown to be an initiating factor for the pulmonary vascular derangement in COPD (12). A study by Lundbäck et al. (13) in 2003 stated that approximately $50 \%$ of smokers may develop COPD. Additionally, it has been noted that nearly $90 \%$ of all COPD patients are smokers (4). Moreover, tobacco use is currently the single most significant and most preventable cause of illness and death in the world, killing approximately 6 million people a year (14). Cigarette smoke is a major source of oxidants, containing an estimated $10^{18}$ spins (electron spin resonance) per gram of tar (particulate matter which is generated by burning tobacco) (4). These radicals are sufficiently stable to be detected by electron spin resonance and may reduce oxygen to $\mathrm{O}_{2}(4)$. Secondly, smoking was found to be the cause of airway and parenchymal inflammation in most COPD patients (4). Airway assessment was performed by sputum analysis, bronchoscopy, biopsy, and lung lavage in COPD patients and suggested that inflammation was a contributing factor to the development of COPD (4).

Oxidative stress, an imbalance between an excess of oxidants and insufficient antioxidant resources, appears to trigger a number of inflammatory, obstructive, and fibrotic lung disorders and contributes to the development of COPD (15). This imbalance is known to play an important role in the pathogenesis of the disease through direct injurious effects, and by involvement in the molecular mechanisms that control lung inflammation (16). The formation from oxygen to superoxide anion $\left(\mathrm{O}_{2}^{-}\right)$, an oxygen radical, is generated primarily by mitochondrial metabolism reactions, arachidonic acid metabolism, and NADPH oxidasedependent processes in phagocytic cells (4). The reaction of $\mathrm{O}_{2}{ }^{-}$and hydrogen peroxide $\left(\mathrm{H}_{2} \mathrm{O}_{2}\right)$ produces the hydroxyl radical $(-\mathrm{OH})$, and when catalyzed by neutrophil 
myeloperoxidase (MPO), $\mathrm{H}_{2} \mathrm{O}_{2}$ and a chloride form hypochlorous acid ( $\left.\mathrm{HOCl}\right)$ (4). Both ${ }^{-} \mathrm{OH}$ and $\mathrm{HOCl}$ are extremely potent oxidants in human bodies(4).

A major consequence of oxidative stress is lipid peroxidation (17). Lipid peroxidation is induced by oxygen in the presence of initiators such as heat, other free radicals, light, photosensitizing pigments, and metal ions (18) which can permanently damage cell membranes, eventually leading to cell death (17). Lipid oxidation occurs via three reaction pathways: non-enzymatic chain autoxidation mediated by free radicals, non-enzymatic and nonradical photooxidation, and enzymatic oxidation (18). One end product of lipid peroxidation, 4-hydroxy-2-nonenal (HNE) for example, may lead to subsequent pathological consequences such as potentiating oxidative stress through depletion of intracellular glutathione and inducing peroxide production (17). HNE has shown to play a role in airway remodeling through activation of the epidermal growth factor receptor and induction of fibronectin production (17). Lastly, past studies have suggested that HNE may induce cell death of alveolar macrophages in mice (17).

Reactive oxygen species (ROS), such as superoxide anion, hydrogen peroxide, and hydroxyl radicals (17), are generated by inflammatory cells in the lung in response to different stimuli, including tobacco smoke (15). Past studies have found that large numbers of neutrophils and macrophages in the lungs of COPD patients generate ROS in excess such that these patients have higher levels of superoxide anion and hydrogen peroxide release (17). Ciencewicki et al (17). have observed impairment in gene expression of protective mechanisms against oxidants in lung samples of COPD patients along with upregulation of chemokines involved in the inflammatory processes. The authors also observed locally generated 4-HNE modifying protein levels in the airway and alveolar epithelial cells, and 
endothelial cells in individuals with airway obstruction, even interacting with glutathione, thereby reducing cells' antioxidant ability (17).

The human body has several mechanisms to counteract oxidative stress by producing antioxidants, or utilizing externally supplied antioxidants through food and supplements (19). Antioxidants can be categorized as either enzymatic and non-enzymatic antioxidants (20). Plant-based antioxidants, vitamin C, vitamin E, carotenoids, and glutathione, are examples of non-enzymatic antioxidants. Antioxidants have been receiving interest in the research field over the past 30 years. According to Halliwell and Gutteridge (18), an antioxidant is defined as "a substance that, when present at low concentrations compared to those of an oxidizable substrate, significantly delays or prevents oxidation of the substrate." An antioxidant-rich diet came to public attention recently due to its role in human health. Collectively, the Mediterranean diet became the supreme diet to the public.

The Mediterranean diet has been known to have protective effects for respiratory diseases in epidemiological studies. The Mediterranean diet pattern consists of high intake of minimally processed whole plant foods such as fruits, vegetables, bread, cereals, beans, nuts, seeds, red wine, and olive oil, low to moderate consumption of dairy foods, fish, poultry, and red meat (21). Several studies in the past found an inverse association between adherence to the Mediterranean diet and atopy, wheezing, and asthma symptoms among children and adults (22). The typical western diet pattern, on the other hand, consists of high consumptions of refined grains, cured and red meats, sweets and sweetened beverages, fried foods, and high-fat dairy products. This pattern of intake has been associated with increased risk of respiratory-related diseases such as asthma in children (23) and adults (24). 
Additionally, a diet consisting of high-fat foods has been shown to worsen airway inflammation and increase the risk of COPD (25).

Fruits and vegetable intake has been known for its prominent effect in respiratory conditions due to their nutrient profile containing antioxidants, vitamins, minerals, fiber, and phytochemicals (26). In a past study, increased fruit intake over two years was associated with increased $\mathrm{FEV}_{1}$, while another study found that a significant decrease in fruit intake over seven years was associated with decreased FEV 1 (27). Wood et al.(28) conducted an intervention in adults with asthma and found that high consumption of fruits and vegetables for three months was associated with a reduced risk for asthma exacerbation. Two randomized controlled trials conducted in COPD patients observed no effect of a high fruit and vegetable intake on $\mathrm{FEV}_{1}$ over a 12-week period; however, a 3-year study showed an improvement in lung function in high fruit and vegetable group compared to the control group (29). This result suggests that a longer-term intervention is needed to provide a therapeutic effect.

A study conducted by Meteran et al. (30) examined the association between selfreported intake of fruits and vegetables and risk of COPD. The study recruited 12,449 twins, between 40 to 80 years of age, from 2008 to 2011 (30). The questionnaire included lifestyle factors, including smoking status, fruits and vegetable intake, degree of physical activity, and respiratory symptoms. A pre-bronchodilator lung function test was performed using a spirometry test and COPD was diagnosed based on the reference values from the Global Lung Initiative 2012. A logistic regression, including sex, age, and BMI as covariates, showed that smoking was an independent predictor of COPD (30). Furthermore, the study found that increase in number of servings of raw fruits and vegetables was associated with a 
significant decrease in risk of COPD (30). This result suggests that raw fruits and vegetables are superior to cooked or processed fruits and vegetables due to higher antioxidant content.

As mentioned above, antioxidants counteract oxidation by protecting target lipids and other target molecules from oxidation (18). It can prevent lipid peroxidation by hindering ROS formation or scavenging species responsible for oxidation initiation (17). Secondly, antioxidants can intercept radical oxidation propagators or indirectly participate in stopping radical chain propagation, and thus prevent peroxidation of lipids (17). To understand how non-enzymatic antioxidants protects lipids from oxidation, the mechanisms of antioxidant activity must be explained.

Vitamin E, $\alpha$-tocopherol, is an efficient lipid-soluble antioxidant that functions as a 'chain breaker' during lipid peroxidation in cell membranes and several lipid particles such as low-density lipoprotein (LDL) (20). It intercepts lipid peroxyl radicals (-LOO) and terminates the lipid peroxidation chain reaction, hence the name 'chain breaker.' The subsequent tocopherol radical is now relatively stable and insufficiently reactive to initiate lipid peroxidation itself.

Vitamin C, ascorbic acid, is a water-soluble free radical scavenger which regenerates vitamin $\mathrm{E}$ in cell membranes in combination with glutathione (GSH) (20). Vitamin C changes to the ascorbate radical by donating an electron to the lipid radical in order to stabilize it and therefore terminate the lipid peroxidation chain reaction (31).

Carotenoids, some of the most common lipid soluble phytonutrients, are known to scavenge the peroxyl radicals more efficiently as compared to any other ROS (20). Scavenging of these radicals can disrupt the reaction sequence and prevent the damage to cellular lipids (20). Carotenoids are also known to protect cellular membranes and 
lipoproteins against the ROS due to their peroxyl radical scavenging activity, where carotenoids deactivate the peroxyl radicals by reacting with them to form resonance stabilized carbon-centered radical adducts (20).

A study in 2000 analyzed the effects of vitamin C, E, $\beta$-carotene, and selenium intake and used both dietary assessment and serum biomarkers of antioxidant status using data from the Third National Health and Nutrition Examination Survey (32). The survey was conducted from 1988 through 1994 on a nationwide sample of approximately 33,994 participants aged two months and older (32). Along with the intake of dietary antioxidants, the authors included the number of cigarettes currently smoked per day. Multiple linear regression analysis was used to examine the separate and joint effects of the antioxidants on FEV (32). As results, the authors found that each of the dietary and serum antioxidant nutrients was significantly associated with $\mathrm{FEV}_{1}$. Although serum $\beta$-carotene was less positively associated with $\mathrm{FEV}_{1}$ in smokers than non-smokers, serum selenium had a strong positive association with $\mathrm{FEV}_{1}$ in smokers (32).

A study conducted in 1998 investigated the relationship between the intake of vitamin $\mathrm{C}, \mathrm{E}$, and $\beta$-carotene and the presence of respiratory symptoms and lung function as part of the MORGEN study (the Monitoring Project on Risk Factors and Health in the Netherlands) (33). The study sample was randomly selected from a cross-sectional study in the Dutch population on 6,555 adults during 1994 and 1995 (33). After collecting data on dietary intake and respiratory symptoms, logistic regression analysis was used for $\mathrm{FEV}_{1}$ and $\mathrm{FVC}$ rate (33). According to the results, the intake of vitamin $\mathrm{C}$ was not associated with most respiratory symptoms, but subjects with a high intake of vitamin C had a 53mL (95\% CI 23 to 83 ) higher $\mathrm{FEV}_{1}$ and $79 \mathrm{~mL}$ (95\% 42 to 116 ) higher $\mathrm{FVC}$ than those with a low vitamin C intake (33). 
Similarly, vitamin E intake showed no association with most symptoms and pulmonary function. The intake of $\beta$-carotene also showed no association with most symptoms; however, subjects with a high intake of $\beta$-carotene had a $60 \mathrm{~mL}$ (95\% CI 31 to 89) higher $\mathrm{FEV}_{1}$ and $75 \mathrm{~mL}$ ( $95 \% \mathrm{CI} 50$ to 110 ) higher $\mathrm{FVC}$ than those with a low intake of $\beta$-carotene (33). These results showed that a high intake of vitamin $\mathrm{C}$ or $\beta$-carotene may be protective for $\mathrm{FEV}_{1}$ and $\mathrm{FVC}$ compared with a low intake (33).

Similarly, Kentson et al. (15) studied the relationship between oxidative stress, iron homeostasis, and plasma levels of carotenoids in patients. This study involved 66 Caucasians with advanced COPD and 47 healthy control subjects. Overall health, lifestyle, and dietary habits were asked in questionnaires, and lung function tests and blood sampling were performed in both groups (15). Results showed that COPD subjects had significantly lower plasma levels of $\beta$-cryptoxanthin, $\alpha$ - and $\beta$-carotenes, and ate significantly less fruits and vegetables in their diet compared to the control group $(\mathrm{p}<0.05)(15)$. The study also found that a diet with high consumption of fruits, vegetables, whole grains, polyunsaturated fatty acids, nuts, and long-chain omega-3 fats and low consumption of red and processed meat, refined grains, and sugar-sweetened drinks was associated with a lower risk of COPD (15).

Recent data describe the beneficial effects of dietary fiber intake on pulmonary symptoms in adults that are independent of antioxidant intake (34). Studies have shown that a higher consumption of fiber was protective against the development of symptoms of chronic bronchitis (34). Kan et al. (34) further investigated the association of dietary fiber intake with lung function and COPD in 11,897 participants of the Atherosclerosis Risk in Communities (ARIC) study. After adjusting for age, ethnicity, sex, smoking status, BMI, total energy intake, antioxidant (vitamin C, E, and D, and omega fatty acids), and cured meat intake, the 
authors observed a positive associations between lung function and fiber intake from all sources (34). Participants in the higher quintile of total fiber intake had $60.2 \mathrm{ml}$ higher FEV 1 $(p<0.001), 55.2 \mathrm{ml}$ higher FVC $(p=0.001), 0.4 \%$ higher $\mathrm{FEV}_{1} / \mathrm{FVC}$ ratio $(p=0.001)$ compared with those in the lowest quintile (34).

Carotenoids are among the most common natural pigments that are synthesized by plants and microorganisms (35) with $\beta$-carotene as the most prominent. The carotenoids in green leafy vegetables and orange-colored fruits and vegetables are known antioxidants that reduce inflammation and prevent oxidative damage by counteracting the effects of free radicals (36). Hence, carotenoids are known to prevent human diseases, including cardiovascular diseases, cancer, and other chronic diseases (35). There are more than 600 carotenoids identified in nature, but about 30 to 40 are present in a typical human diet. Of these 40 about 20 have been recognized in human blood and tissues. About $90 \%$ of the carotenoids in the diet and the human body is represented by $\beta$-carotene, $\alpha$-carotene, lycopene, lutein, $\beta$-cryptoxanthin, $\alpha$-cryptoxanthin, zeaxanthin, $\gamma$-carotene, neurosporene, $\zeta$-carotene, phytofluene, and phytoene $(35,37)$.

Humans cannot synthesize carotenoids; instead, carotenoids must be consumed from diet (38). The European Prospective Investigation into Cancer and Nutrition (EPIC) study measured the plasma levels of six carotenoids in about 3,000 participants and found the following mean levels: lycopene $0.43-1.32 \mu \mathrm{mol} / \mathrm{L}$, lutein $0.26-0.70 \mu \mathrm{mol} / \mathrm{L}, \beta$-carotene 0.21-0.68 $\mu \mathrm{mol} / \mathrm{L}, \beta$-cryptoxanthin $0.11-0.52 \mu \mathrm{mol} / \mathrm{L}, \alpha$-carotene $0.06-0.32 \mu \mathrm{mol} / \mathrm{L}$, zeaxanthin $0.05-0.13 \mu \mathrm{mol} / \mathrm{L}$ (38). Although carotenoids are present in an array of fruits and vegetables, $\beta$-carotene and $\alpha$-carotene are prominent in yellow-orange vegetables and fruits, 
$\alpha$-cryptoxanthin in orange fruits, lutein in dark green vegetables, and lycopene in tomato and tomato products (35).

The primary benefits of carotenoids differ by their antioxidant potential (38).

Carotenoids are known to be the most effective in physical and chemical suppression of singlet oxygen $\left({ }^{1} \mathrm{O}_{2}\right)$, as well as potent scavengers of other ROS (37). Hence, carotenoids are acknowledged to play a protective role in many ROS-mediated disorders like cardiovascular diseases, several types of cancer, neurological disorders, and photosensitive or eye-related disorders (37). Specific carotenoids can act through different mechanisms. For instance, $\beta$-carotene has added benefits due to its ability to be converted to vitamin A, while lutein and zeaxanthin absorb specific wavelengths of light which could help protect the eyes and may also reduce cognitive decline (38). Lycopene is known for its role in preventing cancer and heart diseases (35). Lastly, lutein is known to be beneficial in maternal and infant health (38).

Like many other nutrients, bioavailability and absorption of carotenoids depend on several factors, including food processing and cooking. Once consumed, carotenoids are absorbed into the gastrointestinal mucosal cells and appear unchanged in the circulation and tissues (35). The carotenoids are absorbed by passive diffusion after being incorporated into the micelles that are formed by dietary fat and bile acids in the intestine (35). The micellular carotenoids are then incorporated into the chylomicrons and released through the lymphatic system and also into lipoproteins in the liver where carotenoids are then released into the blood stream (35). The distributed carotenoids are incorporated in very low-density lipoproteins (VLDL); thus, the resulting LDL exhibit the highest concentration of carotenes in plasma (37). 
Blood concentrations of carotenoids are known to be a reliable biological marker for fruits and vegetable consumption (39). The major serum carotenoids are $\alpha$-carotene, $\beta$-carotene, $\beta$-cryptoxanthin, lycopene, and zeaxanthin. The concentration of these carotenoids in human serum and tissues are highly variable and likely to depend on different factors such as food sources, the efficiency of absorption, and amount of fat in the diet (39). The serum carotenoid concentration after a single dose peak at 24 to 48 hours post dose (39). Total serum carotenoid content is not an exclusive measure of newly absorbed carotenoids. Therefore, the increase in carotenoids in the triglyceride-rich lipoprotein fraction - earliest postprandial serum appearance of carotenoids, is used to quantify carotenoid absorption (39). Prior epidemiological studies found a positive association between higher dietary intake and tissue concentration of carotenoids and lower risk of chronic diseases (35). Studies suggest that the antioxidant properties of carotenoids, especially $\alpha$ - and $\beta$-carotene and $\beta$-cryptoxanthin, are the main mechanism in disease prevention (35). Recent studies also showed that carotenoids may facilitate their effects through other mechanisms such as gap junction communication, cell growth regulation, modulating gene expression, immune response and as modulators of Phase I and II drug metabolizing enzymes (35). Carotenoids are also known to participate in the regulation of the cell cycle apoptosis and the modulation of various types of receptors or adhesion molecules and many other physiologically significant processes (37).

A meta-analysis of prospective studies evaluated the associations between $\beta$-carotene intake or circulating $\beta$-carotene levels on the risk of all-cause mortality in general healthy populations (36). The study observed a favorable impact of dietary or circulating $\beta$-carotene on the risk of all-cause mortality among the general population in observational studies. The 
authors explained that $\beta$-carotene in natural forms may reduce the risk of mortality in humans through several mechanisms. $\beta$-carotene can play an important role in its pro-vitamin A activity which is essential to the human body for regular tissue differentiation, organogenesis, immune competence, and for maintaining normal vision. $\beta$-carotene may exert physiological action by both antioxidant and pro-oxidant effects, which are dependent on the oxygen tension in the human body. With a low oxygen tension, $\beta$-carotene and other antioxidants can act synergistically as an effective radical-scavenging antioxidant in biological membranes. Moreover, $\beta$-carotene may enhance immune cell function to play a significant role in the prevention of chronic diseases (40).

Past studies have reported that smokers have lower plasma carotenoid concentrations compared to non-smokers, in fact, the greater intensity of smoking was associated with decrease in serum carotenoid concentrations (39). Tobacco smoke is highly oxidative, and the gas phase of tobacco smoke has been shown to destroy carotenoids in in vitro studies of human plasma (39). Consequently, both smoke and gas-phase smoke oxidize carotenoids to carbonyls, epoxides, and nitro derivatives, which contribute to the reduction in circulating levels (39). Although smoking status increases the need for a higher intake of carotenoids to achieve an optimal plasma concentration of carotenoids, supplementation of carotenoids are generally not recommended as carotene supplements have been suggested as causing adverse effects in smokers (39).

An association of vitamin A status and the rate of COPD was observed in a crosssectional study. The purpose of this study was to examine if vitamin A deficiency is associated with COPD (41). The study assessed healthy nonsmokers and smokers and COPD patients. Sixty-five adult male subjects aged between 43 and 74 were recruited in the study. 
Data for smoking habits, pulmonary function tests, and energy-protein status were collected (41). Serum concentrations of retinyl esters, retinol, retinol-binding protein, and transthyretin and relative dose responses were measured (41). A significantly lower serum concentration of retinol was observed in the COPD groups (41).

It is evident that oxidative stress contributes to airflow limitation, making the association between antioxidants and pulmonary function more apparent in individuals with COPD compared to general healthy population. Because of the higher needs of antioxidants among individuals with limited pulmonary function, the antioxidant status may be important in the progression of pulmonary diseases (42). Ochs-Balcom et al. (42) studied the association between antioxidant nutrients and oxidative stress biomarkers with pulmonary function in persons with chronic airflow limitation. The authors hypothesized that antioxidant vitamins would be positively related to pulmonary function, while oxidative stress markers may be negatively associated with pulmonary function in patients with a limited airflow.

The study participants were tested for pulmonary function, and fat-soluble vitamins in serum were measured using a high-pressure liquid chromatography system. Nutrient intake was assessed over the 12-month period using the self-administered 100 item food frequency questionnaire. Then, individual mean daily nutrient intakes were calculated (42). Of the 218 study participants, $66 \%$ were current and former cigarette smokers with a mean of 24.4 packyears of smoking. Adjusting for covariates, multiple linear regression analysis showed that serum $\beta$-carotene, lutein, zeaxanthin, and retinol, and dietary $\beta$-carotene, $\beta$-cryptoxanthin, lutein, zeaxanthin, lycopene, and vitamin $\mathrm{C}$ were positively associated with FVC percentage $(p<0.05)(42)$. Partial correlation coefficients $(r)$ among serum vitamins ranged from 0.16 to 0.44, where the strongest correlation was found among $\beta$-cryptoxanthin and lutein/zeaxanthin 
$(r=0.43, p<0.001)$ and vitamin $\mathrm{E}$ with $\beta$-carotene $(r=0.42, p<0.001)(42)$. Of the dietary vitamins, $\beta$-carotene and lutein $(r=0.70, p<0.001)$ had the strongest correlation (42). The authors of this study suggested that $\beta$-cryptoxanthin and lutein/zeaxanthin are more strongly associated with pulmonary function than $\beta$-carotene which strengthens the evidence that the antioxidant/oxidant balance is associated with pulmonary function and that carotenoids may play an important role (42).

A study performed by Semba et al. (8) examined the relationship between the major serum carotenoids, $\alpha$-carotene, $\beta$-carotene, $\beta$-cryptoxanthin, lutein/zeaxanthin, and lycopene, with pulmonary function. The study hypothesized that serum carotenoids were associated with pulmonary function in older women. To address this hypothesis, the researchers measured serum carotenoids and assessed pulmonary function in 1,002 women, aged 65 years and older, who participated in the Women's Health and Aging Study I (WHAS I) (8). Results showed a positive association between higher serum $\alpha$-carotene and $\beta$-carotene concentrations and $\mathrm{FEV}_{1}$ and $\left.\mathrm{FVC} \mathrm{(all} p<0.05\right)$ in separate multivariate linear regression models adjusting for age, race, education, cognition, anemia, inflammation, and chronic diseases (8). Total serum carotenoids were associated with $\mathrm{FEV}_{1}(p=0.08)$ and FVC $(p=0.06)$, respectively, in similar models; however, the study did not find an association between $\beta$-cryptoxanthin, lutein/zeaxanthin, and lycopene, and $\mathrm{FEV}_{1}$ or $\mathrm{FVC} \mathrm{(8).}$

Grievink et al. (43) designed a study to observe the association between serum carotenoids, $\alpha$-tocopherol, and lung function among non-institutionalized Dutch subjects ages 65 to 85 years. Multiple linear regression analysis was performed adjusting for age, gender, height, and cigarette-years of smoking. Based on $\mathrm{FEV}_{1}$ and $\mathrm{FVC}$ values, $47 \%$ of the study participants had airway obstruction (43). The authors reported that $\mathrm{FEV}_{1}$ was highly 
correlated with age, height, physical activity score, and serum cholesterol, but not with BMI (43). Lower $\mathrm{FEV}_{1}$ and $\mathrm{FVC}$ were observed among former and current smokers compared to non-smokers (43). The study found significantly positive trends between $\alpha$-carotene, $\beta$-carotene, lycopene, and $\mathrm{FEV}_{1}(p<0.05)$ and between $\alpha$-carotene, $\beta$-carotene, and FVC $(p<0.05)$ (43). Although lycopene was associated with $\mathrm{FEV}_{1}$, it was not significantly associated with FVC (43). A possible reason, as the authors noted, could be that most carotenoids were measured in serum and in lung tissue, in which the proportion of lycopene in the lung tissue was slightly lower, and $\alpha$-carotene was higher compared with the proportion of the other carotenoids in serum levels (43). Therefore, the concentration of lycopene and $\alpha$-carotene in the blood may not represent the actual value in lung tissue (43).

A study examined the relationship between lung cancer risk and intakes of the five carotenoids in two large cohorts. Baseline questionnaires asked participants to report their average frequency of intake over the previous year of a specified serving size of specific carotenoid containing foods (44). Carotenoid intakes were derived from the reported consumption of fruit and vegetables on the questionnaire (44). Smoking status and history and specified medical conditions were assessed at baseline and in all subsequent questionnaires in both cohorts. After the 12-year follow-up period, the study found that $\alpha$-carotene and lycopene intakes were significantly associated with a lower risk of lung cancer $(p<0.05)$; the association with $\beta$-carotene, lutein, and $\beta$-cryptoxanthin intakes were inverse but not significant (44). Additionally, lung cancer risk was significantly lower in subjects who consumed a diet high in a variety of carotenoids (RR: $0.68 ; 95 \%$ CI: $0.49,0.94$ for highest compared with lowest total carotenoid score category) (44). 
Lycopene is the most abundant carotenoid in tomatoes and tomato products such as ketchup and pasta sauce. Epidemiological studies show that high intake of tomatoes and tomato products containing high levels of lycopene, exhibit a lower risk of lung cancer (31). Lycopene is also known to have anti-tumoral activity in lung tumorigenesis. The high number of conjugated double bonds in lycopene endows it the singlet oxygen quenching ability as compared to vitamin E or $\beta$-carotene (20). The chemical structure of lycopene is an electron-rich system making it unstable and highly reactive towards oxygen and free radicals (31). This reactivity of lycopene is the base of its antioxidant activity in biological systems. Additionally, lycopene may exert modulatory action on cancer by up-regulating antioxidant response element leading to the synthesis of cytoprotective enzymes, enhancing intercellular gap junction communication, modulating the hormonal, inflammatory and immune system, and metabolic pathways (31).

An animal study investigated whether lycopene reverses long-term lung damage caused by cigarette smoke exposure. The authors hypothesized that administration of lycopene would repair lung damage in emphysema induced by cigarette smoke exposure. In the study, mice were divided into five groups: control group, oil group, group exposed to cigarette smoke, group administered with $25 \mathrm{mg}$ of lycopene and exposed to cigarette smoke, and groups administered with 50mg of lycopene and exposed to cigarette smoke. Within the two groups that were given a dose of lycopene, half was exposed to 60 days of cigarette smoke, and the other was not. The study found an increased number of circulating inflammatory cells and increased levels of inflammatory cytokines $(p<0.05)$ in the group that was exposed to cigarette smoke (14). In contrast, there was a reduction in the number of total leukocytes, reduced cytokines, and improved pulmonary emphysema among the lycopene 
administered mice as determined by the number of macrophages, neutrophils, lymphocytes, and inflammatory cytokines (14). In summary, lycopene promoted a reduction in the number of total leukocytes and improved pulmonary emphysema. The authors also observed minimized redox processes due to a decrease in lipid peroxidation and DNA damage and an increase in the activities of superoxide dismutase (SOD), catalase (CAT), and GSH content (14).

A randomized clinical trial in 2012 observed the effect of lycopene on oxidant stress and pulmonary function tests (PFT) in patients with COPD (45). Fifteen clinically stable COPD patients and 15 healthy non-smoker control subjects were divided into two groups. One group received oral placebo, and the other group received $20 \mathrm{mg}$ lycopene supplement daily for four months in addition to their standardized therapy. Their baseline spirometry, plasma malondialdehyde (MDA), SOD, CAT, interleukin (IL)-6, IL-1 $\beta$, and tumor necrosis factor- $\alpha$ levels were measured in serum (45). At the beginning of the trial, the COPD group had higher baseline mean MDA, IL-6, IL-1 $\beta$, and TNF- $\alpha$ levels and lower SOD and CAT levels compared to controls (45). After four months of lycopene supplementation, however, significant increases in mean SOD, and CAT levels $(p<0.001)$, and significant decreases in mean MDA, IL-6, IL-b, and TNF-a levels $(p=0.001, p<0.001, p=0.002, p<0.001$, respectively) were observed in the lycopene-supplemented patients, suggesting that lycopene supplementation may have favorable effects on oxidant-antioxidant balance in patients with COPD (45). The study found no significant increase in FEV1/FVC ratio (45).

The Military and Veteran's Health Study examined the intake and serum levels of nutrients previously associated with lung health in a population of veterans with COPD (46). A secondary analysis of data was obtained from a cross-sectional study of agricultural 
exposures and COPD in veterans. A subject was considered to be a smoker if they had smoked more than 100 cigarettes in their lifetime. A food frequency questionnaire (FFQ) was used to determine absolute nutrient intake values from foods and supplements. Serum levels of the five carotenoids were measured. For statistical analysis, descriptive statistics were calculated for all variables, and a Spearman correlation coefficient was used to look at the association between serum carotenoid levels and the lung function measures $\left(\mathrm{FEV}_{1} / \mathrm{FVC}\right.$ ratio). The study documented lower serum levels of $\beta$-cryptoxanthin and carotenes among smokers (46). Additionally, there was a correlation between dietary $\beta$-cryptoxanthin and the high serum level of this compound with the decreased rates of COPD (46).

Rautalahti et al. (47) studied the effect of $\alpha$-tocopherol and $\beta$-carotene supplementation on COPD symptoms. This study aimed to investigate whether supplementation with $\alpha$-tocopherol and $\beta$-carotene could affect the recurrence and incidence of COPD-related symptoms and whether dietary intake and serum levels of those substances were related to the prevalence of COPD symptoms in a cohort of smokers (47). Subjects were current smokers of at least five cigarettes daily and had smoked for a median of 36 years. Results indicated that supplementation with $\alpha$-tocopherol or $\beta$-carotene for five to eight years did not prevent the development of COPD, but a diet rich in carotenoids offered some protection even among elderly, long-term smokers (47). There are possible explanations for why the supplementation of carotenoids did not offer protection against free radicals: the antioxidant supplementations may have been given too late or for too short a period of time, or the high levels of antioxidants may have disturbed a balance between oxidants and various antioxidants (47). 
Studies in the past have documented a potentially harmful effect of carotenoids on lung outcomes with an increased risk for lung cancer and mortality among smokers (15). Studies found that supplementation of high doses of $\beta$-carotene increased the risk of lung cancer in smokers (48). The National Institute of Health explained several reasons for this. First, the beneficial health effects of a diet high in vegetables and fruits or other antioxidantrich foods may actually be caused by other substances present in the same foods, other dietary factors, or other lifestyle choices rather than antioxidants. The effects of the large supplemental doses of antioxidants used in the studies may be different from those of the smaller amounts of antioxidants normally consumed in foods. Lastly, the effects of the different chemical composition of antioxidants in foods and those in supplements may have influenced the result (48).

The results of the studies that observed the role of $\beta$-carotene in airway obstruction are inconsistent. Several prospective studies found a weak inverse association between dietary carotenoids and adult-onset asthma, and some studies found no association between baseline $\beta$-carotene intake and COPD mortality (6). These contradictory results may be due to measurement error, the choice of the dietary instrument, and the differences in outcome assessment.

These conflicting results, coupled with the current lack of effective treatments for COPD, indicate a need for further research into possible preventative measures. The present study aims to analyze the association between dietary intake of carotenoids and pulmonary function outcomes among participants of the Atherosclerosis Risk in Communities (ARIC) study. Based on the previous results from similar studies, it is hypothesized that there is an inverse relationship between the intake of carotenoids and carotenoid-rich foods and the rates 
of COPD, with higher intakes of carotenoids and carotenoid-rich foods being associated with lower rates of COPD. 


\title{
Chapter Two
}

\begin{abstract}
Background: Chronic obstructive pulmonary disease (COPD) is an inflammatory disease causing more than three million deaths annually around the world. Previous studies have shown an increased incidence of COPD among smokers. Studies also have shown antioxidant nutrients such as carotenoids, have been associated with lower rates of COPD. Objective: To investigate if the consumption of carotenoids and carotenoid-rich foods is associated with higher pulmonary function.
\end{abstract}

Methods: Data were taken from the Atherosclerosis Risk in Communities (ARIC) study, which included approximately 15,000 individuals aged 45-64 years at baseline. Dietary intake of carotenoids and carotenoid-rich foods were assessed. Total carotenoid intake was calculated by summing five specific carotenoids: $\alpha$-carotene, $\beta$-carotene, $\beta$-cryptoxanthin, lycopene, and lutein/zeaxanthin. Pulmonary function was evaluated as the ratio of forced expiratory volume in one second $\left[\mathrm{FEV}_{1}\right]$ and forced vital capacity $[\mathrm{FVC}]$. Linear regression analysis was used to assess the association between the intakes of carotenoids and carotenoid-rich foods. Significance level was $p<.05$.

Results: A positive association between the total carotenoid intake with pulmonary function was only marginally significant; however, $\alpha$-carotene, $\beta$-carotene, and $\beta$-cryptoxanthin were positively ( $p=.001, p=.003, p=.007$, respectively) associated with $\mathrm{FEV}_{1} / \mathrm{FVC}$ ratio in study participants. Food sources of these pro-vitamin A carotenes were also $(p=.008)$ positively associated with $\mathrm{FEV}_{1} / \mathrm{FVC}$ ratio.

Conclusions: This study suggests a possible role for certain provitamin A carotenes, and their associated foods in pulmonary health.

Keywords: diet; carotenoids; pulmonary; pulmonary function; lung; COPD 


\section{Introduction}

Chronic obstructive pulmonary disease (COPD) is the fourth leading cause of death in the world and is projected to be the third leading cause of death by 2020 (1). According to the Global Initiative for Chronic Obstructive Lung Disease (1), more than three million people died of COPD in 2012, which is six percent of all deaths globally. Based on large scale epidemiological studies, the number of COPD cases was estimated to be 384 million in 2010, with a global prevalence of 11.7\% (95 CI 8.4\%-15.0\%) (1).

COPD is an inflammatory disease leading to obstructive lung airways and airflow limitations (2) that interferes with normal breathing (3). A COPD diagnosis is considered in any patient who has symptoms of a chronic cough, sputum production, dyspnea, and a history of exposure to risk factors for the disease (4). The diagnosis is confirmed by a spirometry test, which measures how fast air can move out of the lungs and how deeply a person can breathe (5). More specifically, the ratio of the forced expiratory volume in 1 second $\left(\mathrm{FEV}_{1}\right)$ to the total vital capacity $(\mathrm{FVC})$ is used to define the severity of the disease (6). COPD is classified by a $\mathrm{FEV}_{1} / \mathrm{FVC}$ ratio of less than $70 \%$ or by an $\mathrm{FEV}_{1}$ less than $80 \%$ of that predicted by age, sex, ethnicity, and standing height (7).

The development of COPD is mainly due to biological factors such as inflammation and oxidative stress caused by free radicals, and inadequate intake of antioxidant-rich foods (8). Augmented pollution from rapid industrialization and increases in the smoking rates worldwide also increase risk for developing COPD (9). According to Scanlon et al. (10), risk factors that contribute to the development of COPD include tobacco smoking, poor initial lung function, advanced age, male sex, childhood respiratory illness, occupational respiratory exposures, air pollution, low educational attainment or socioeconomic status, and other familial factors. 
Carotenoids are among the most common natural pigments that are synthesized by plants and microorganisms (35) with $\beta$-carotene as the most prominent. The carotenoids in green leafy vegetables and orange-colored fruits and vegetables are known antioxidants that reduce inflammation and prevent oxidative damage by counteracting the effects of free radicals (36). Recent studies also showed that carotenoids may facilitate their effects through other mechanisms such as gap junction communication, cell growth regulation, modulating gene expression, immune response and as modulators of Phase I and II drug metabolizing enzymes (35).

A study performed by Semba et al. (8) examined the relationship between the major serum carotenoids, $\alpha$-carotene, $\beta$-carotene, $\beta$-cryptoxanthin, lutein/zeaxanthin, and lycopene, with pulmonary function. Results showed a positive association between higher serum $\alpha$-carotene and $\beta$-carotene concentrations and $\mathrm{FEV}_{1}$ and $\mathrm{FVC}(p<0.05)$ in separate multivariate linear regression models (8).

In contrast, some studies observed no association between carotenoid intake and COPD risk. A community-based Korean Genome Epidemiology Study (KoGES) study found a decreased risk of COPD with increase in the dietary vitamin A and vitamin $\mathrm{E}$ intake in men (49). Furthermore, the lung function was significantly improved with increase in vitamin C and $\mathrm{E}$ intake (49). However, the study found no significant association in baseline vitamin A or $\beta$-carotene intake and the COPD risk, suggesting a nonbeneficial effect of $\beta$-carotene in human lung (49).

These conflicting results, coupled with the current lack of effective treatments for COPD, indicate a need for further research into possible preventative measures. The present study aims to further analyze the association between dietary intake of carotenoids and 
carotenoid-rich foods and pulmonary function outcomes among participants of the Atherosclerosis Risk in Communities (ARIC) study. Based on the previous results from similar studies, it is hypothesized that there is a positive relationship between the intake of carotenoids and carotenoid-rich foods and the $\mathrm{FEV}_{1} / \mathrm{FVC}$ ratio.

\section{Materials and Methods}

\section{Study Population}

The Atherosclerosis Risk in Communities Study (ARIC), sponsored by the National Heart, Lung, and Blood Institute (NHLBI), is a large prospective epidemiologic study designed to examine the risk factors for and clinical outcomes of atherosclerosis by race, gender, and location. The study randomly selected and recruited approximately 15,000 individuals aged 45-64 years from four communities in the U.S.: Forsyth County, North Carolina; Washington County, Maryland; the suburbs of Minneapolis, MN; and, the City of Jackson, Mississippi (50). A total of 15,792 participants received extensive examinations, including medical, social, and demographic data. Follow-up occurred semi-annually by telephone, to assess health status of the cohort, and reexamination occurred every three years with the baseline visit taking place in 1987-89 $(51,50)$.

\section{Dietary Intake and Carotenoid Level}

The participants' average dietary intake data were collected by an interviewer using a 66-item, semiquantitative food frequency questionnaire (FFQ), a modified version of the 61-item questionnaire developed by Walter Willett (50). The major modification was the inclusion of an interviewer trained to use a standardized procedure for administrating the dietary questionnaire (50). Participants were asked how often on average they had consumed a specified portion size of each food during the preceding year with nine response categories 
available. Daily nutrient intake was calculated by multiplying the nutrient content of the specified portion of each food item by the frequency of its daily consumption and summing over all items. For this study, daily intakes of the five carotenoids, $\alpha$ - and $\beta$-carotene, $\beta$-cryptoxanthin, lycopene, and lutein/zeaxanthin, were calculated from the ARIC FFQ, then, the total carotenoids were calculated. Intake of carotenoid-rich foods was calculated by adding the number of servings consumed daily of fruits and vegetables that are rich in each carotenoid.

\section{Pulmonary Function}

Pulmonary Function Assessment is one of the series of protocols and manuals of operation for the ARIC study. The pulmonary function was measured at visit 1 and 2 using Collins Survey II volume displacement spirometer which is connected to an IBM PC/XT computer (52). A spirometry summary and interpretation from the IBM Proprinter was created for each ARIC study participant (52). A technician coached the participant both to maximal inspiration and to maximal expiration. Participants made five attempts to obtain three measurements that were considered acceptable. The technician also judged the acceptability and quality of the subject's effort. As a subject blow into the spirometer, the spirogram paper displays a volume-time tracing while the computer displays flow-volume curves for operator assessment. At the same time, the computer makes multiple quality measurements of each maneuver. A message then is displayed when at least two out of three tests are reproducible (52). The computer then prints a summary of the subject's results from the data file at the end of each session and then stores the raw data from each test in the file generated for that subject. For the present analysis, pulmonary function was evaluated as a 
ratio of the forced expiratory volume in one second $\left[\mathrm{FEV}_{1}\right]$ and forced vital capacity $[\mathrm{FVC}]$ and diagnosis of COPD was evaluated as a $\mathrm{FEV}_{1} / \mathrm{FVC}$ ratio of less than $70 \%$.

\section{Demographic and Other Confounding Variables}

Other information, including age, height, weight, gender, ethnicity, energy intake, smoking status, education level, physical activity level, and medical and personal information were collected during the clinical examination and home interview at baseline (50). Experienced and trained interviewers were employed to conduct data collection activities. Anthropometric measurements were taken by technicians. Results for standing body height was recorded to the centimeter, rounding down. The participant's body weight was recoded to the pound, rounding down (50). Self-reported smoking status was classified as current smoker, non-smoker, or past-smoker (50). Cigarette-years of smoking were assessed. Body mass index (BMI) was calculated as weight $(\mathrm{kg}) /$ height $(\mathrm{m})^{2}$. Education was recorded as a 3-step scale, basic education or less than a high school degree, intermediate education with a high school degree but less than a 4-year degree, or advanced education with a 4-year degree or beyond. Physical activity level was quantified as tertiles of the sum of work, sports, and leisure time activities.

\section{Statistics}

Study participants who met the following criteria were excluded: ethnicity other than African American or white; missing data on spirometry or intake of carotenoids; $\mathrm{FVC}_{1} / \mathrm{FEV}$ ratio of less than $40 \%$ or more than $95 \%$; carotenoids intake of more than $40 \mathrm{mg}$ per day; and missing or incomplete information for covariates, including smoking status and total energy intake. The final study sample consisted of 13,825 adults. 
Linear regression models of total dietary intake of carotenoids versus pulmonary function were created, controlling for age, sex, ethnicity, education level (3 levels), standing height (centimeters), BMI, smoking status, cigarette-years of smoking, total energy intake, total physical activity level, vitamin C intake, and total cholesterol level.

All statistical analysis was performed using IBM SPSS Statistics, version 24 (IBM Corp., Armonk, NY).

\section{Results}

A total of 13,825 participants were assessed for inclusion in the present study. Study participants were female $(55 \%, n=7,832)$, black $(25 \%, n=3,593)$ with advanced level education (35.4\%). Ages at baseline ranged from 44 to 66 years. Mean BMI was $27.7 \pm 5.3$. Current smokers had smoked an average of $639.7 \pm 444.2$ cigarette-years. Table 1 shows the characteristics of the study cohort by smoking status.

Overall $\mathrm{FEV}_{1} / \mathrm{FVC}$ ratio in this cohort was low; never smokers, past smokers, and current smokers had an average percentage of $76.9 \pm 6.0,74.1 \pm 7.4$, and $71.4 \pm 8.7$ respectively (Table 1). Average total carotenoid intake was $8.9 \pm 6.5 \mathrm{mg}$ per day.

Linear regression comparing total carotenoid intake alone and $\mathrm{FEV}_{1} / \mathrm{FVC}$ ratio at visit 1 showed a significant univariate association $(p=0.016)$. An analysis controlling for demographic variables including black ethnicity, female gender, and age attenuated this association with the $\mathrm{FEV}_{1} / \mathrm{FVC}$ ratio $(p=0.057)$. Finally, an analysis controlling for the demographic variables and other important covariates including education level, smoking status, cigarette-years of smoking, BMI, physical activity level, total energy intake, and standing height showed a marginal significant association $(p=0.038)$ (Table 2$)$. A similar 
marginal association between $\mathrm{FEV}_{1} / \mathrm{FVC}$ ratio and carotenoid intake was found in all of the smoking subgroups.

Each carotenoid was analyzed separately through a multivariate linear regression. $\mathrm{FEV}_{1} / \mathrm{FVC}$ ratio showed a promising positive association with $\alpha$-carotene, $\beta$-carotene, and $\beta$-cryptoxanthin. Lycopene and lutein/zeaxanthin showed no significant association with $\mathrm{FEV}_{1} / \mathrm{FVC}$ ratio (Table 3 ). These results show possibly differing roles of various dietary carotenes in lung health.

After controlling for vitamin $\mathrm{C}$, both $\alpha$-carotene, $\beta$-carotene, and $\beta$-cryptoxanthin had a significant association with $\mathrm{FEV}_{1} / \mathrm{FVC}$ ratio $(p<0.001$ and $p<0.008$, respectively), which shows that both carotenes are highly associated with a better pulmonary function independent of the other important antioxidant vitamin source in fruits and vegetables (Table 3).

Additionally, total servings of fruits and vegetables that are high in $\alpha$-carotene, $\beta$ - carotene, and $\beta$-cryptoxanthin, such as oranges, orange juice, peaches, carrots, winter squashes, and sweet potatoes, were analyzed (Table 4) to see their association with $\mathrm{FEV}_{1} / \mathrm{FVC}$ ratio. Orange- and yellow-colored fruits and vegetables intakes were observed to be significantly associated with $\mathrm{FEV}_{1} / \mathrm{FVC}$ ratio $(p=0.008)$ (Table 5). In contrast, intake of fruits and vegetables that are high in lycopene and lutein/zeaxanthin, such as cabbage, tomatoes, greens, corns, peas and lima beans, and pasta, had no association on pulmonary function. The ARIC study participants ate an average of $2.0 \pm 1.5$ servings of fruits and $1.4 \pm 0.9$ servings of vegetables daily.

\section{Discussion}

The present study shows marginally significant association between the total carotenoid intake and pulmonary function, which rejects the null hypothesis. We found 
differences among the carotenoids, with a higher intake of $\alpha$-carotene, $\beta$-carotene, and $\beta$-cryptoxanthin being positively associated with pulmonary function in adults, as expressed by $\mathrm{FEV}_{1} / \mathrm{FVC}$. Foods rich in these carotenoids were also found to be associated with a higher $\mathrm{FEV}_{1} / \mathrm{FVC}$ ratio.

These findings are consistent with past studies that have found a significant association between both $\alpha$ - and $\beta$-carotenes and lung function $(8,43)$. Although past studies did not show a significant association between $\beta$-cryptoxanthin and $\mathrm{FEV}_{1}$ or $\mathrm{FVC}$, they found a marginal association between lycopene and lung function (43) similar to the present study. The present study assessed dietary intake of carotenoid-rich foods instead of serum carotenoid levels which could have led to different results than the past studies.

The present study considered all confounding factors, such as sex, age, education level, BMI, total calorie intake, height, smoking status, pack-years of cigarette smoking, physical activity level, and total serum cholesterol level, in the association between carotenoid-rich food intake and $\mathrm{FEV}_{1}$ or $\mathrm{FVC}$ ratio. A significant association was observed after taking these confounders into account. However, there may have been measurement error in the confounding variables in which residual bias might have occurred.

The present study observed significant associations of certain carotenoids in lung function. A past study that looked at dietary total carotene in relation to lung function stated that different carotenoids might have individual effect on lung physiology (53). In an animal study, three months of $\beta$-cryptoxanthin supplementation substantially reduced smokeelevated inflammation in lung tissues (54). The authors explained that the beneficial effects of $\beta$-cryptoxanthin on lung function observed in this study were not due to its pro-vitamin activity but due to its anti-inflammatory and anti-oxidative DNA effects in the lungs (54). 
In contrast to the above studies, Schünemann et al. (55) found the strongest correlation between intake of lutein/zeaxanthin and pulmonary function, significantly with FVC. This population-based study examined the five carotenoids and found no association between $\beta$-cryptoxanthin intake. The authors explained that this lack of an association may be the result of the difficulties associated with accurately measuring $\beta$-cryptoxanthin in the $\operatorname{diet}(55)$.

According to USDA Food Composition Databases (56), foods that are high in $\alpha$-carotene include carrots and winter squashes. $\beta$-carotene is rich in sweet potatoes, carrots, and greens such as spinach. $\beta$-cryptoxanthin is rich in winter squashes and oranges. Carrots, sweet potatoes and winter squashes are abundant in all $\alpha$-carotene, $\beta$-carotene, and $\beta$-cryptoxanthin. Statistical correlations in the ARIC dataset indicated that all fruits, vegetables, legumes, and nuts were significantly associated with $\alpha$-carotene, $\beta$-carotene, and $\beta$-cryptoxanthin. More specifically, carrots had the highest association with both $\alpha$-carotene and $\beta$-carotene, and oranges had the highest association with $\beta$-cryptoxanthin. Processed foods such as commercially baked goods, meats, eggs, dairy, and wine had limited or no associations with these. The consumption of fruits and vegetables rich in $\alpha$-carotene, $\beta$-carotene, and those rich in $\beta$-cryptoxanthin and lycopene and lutein/zeaxanthin was analyzed across smoking status (Table 5). Study participants ate about 1.5 servings of carotene-rich fruits and vegetables and 1 serving of lutein/zeaxanthin-rich fruits and vegetables daily. Among the three groups, never smokers ate more servings of carotenoidrich fruits and vegetables.

Total servings of pasta among the ARIC study participants were calculated since most lycopene is consumed from tomato products such as pasta sauce. Certainly, pasta 
consumption was correlated with lycopene intake; however, it was not significantly associated with pulmonary function in this cohort.

There are possible explanations for these results. Lycopene is mostly consumed from tomatoes and tomato products including pizza and pasta which are not considered vegetables. For which, higher consumption of pizza and tomato-based pasta dishes may not have a positive effect in pulmonary function.

Lutein/zeaxanthin comes mostly from green vegetables such as spinach, kale, and lettuce. Intake of lutein/zeaxanthin-rich fruits and vegetables were not significantly associated with pulmonary function.

Other antioxidant nutrients are thought to be protective of lung function in adults. Vitamin C, vitamin E, flavonoids, and selenium are examples of these antioxidants. These are present in a variety of fruits and vegetables that are also high in $\alpha$-carotene, $\beta$-carotene, and $\beta$-cryptoxanthin. Thus, we cannot conclude that carotenoids are the only nutrients playing a role in lung function. In the present study, adjusting for vitamin $\mathrm{C}$ intake, however, still found significant associations between both $\alpha$ - and $\beta$-carotenes and $\mathrm{FEV}_{1} / \mathrm{FVC}$ ratio.

A past study suggested that improving the diet, by increasing fresh fruits and vegetable consumption, rather than of vitamin supplements, will be beneficial in protecting airway diseases (57). As five carotenoids can be consumed from food sources, including carrots, sweet potatoes, tomatoes, etc., consumption of fruits and vegetables as a whole may be the best way to increase carotenoids intake along with the other antioxidant nutrients. A study examined the association of self-reported intake of fruit and vegetables and COPD in a population-based twin study and found a significant frequency-dependent association between fruit and vegetable intake and risk of COPD (30). The study suggested that a low 
intake of fruits and vegetables is associated with an increased risk of COPD (30). This information, coupled with the present study results, shows that higher intake of fruits and vegetables, especially those rich in carotenoids, are beneficial for lung health.

One of the strengths of the present study was the large population-based sample of ARIC study participants. The ARIC study included adults who are at higher risk of decreased pulmonary function. The study also included measurement of all five major dietary carotenoids and spirometry measurements of lung function. Lastly, the ARIC study had relevant possible cofactors such as smoking status, physical activity level, BMI, and total energy intake.

This study has several limitations. The present study was a cross-sectional study which assumes that the assessed diet had been in place for some time so as to predispose for the lung condition. It is possible that the covariate intake of other antioxidant nutrients such as vitamin $\mathrm{C}$, vitamin $\mathrm{E}$, and selenium may confound the apparent carotenoid association. The ARIC study assessed nutrient intakes using the FFQ method, which may have caused limitations such as measurement error in the portion sizes and inaccuracies that resulted from limited food choices. Another limitation is that the ARIC study only recruited adults older than 45 , in which case the results cannot necessarily be extrapolated to a younger population. Lastly, we did not control for the geographical locations of the four ARIC study centers which may have affected the lung function of the study participants depending on the environment.

In conclusion, although this present study showed only a marginal association between lung function and total carotenoid intake, a promising association with pro-vitamin A carotenoids, including $\alpha$-carotene, $\beta$-carotene, and $\beta$-cryptoxanthin, was seen with 
$\mathrm{FEV}_{1} / \mathrm{FVC}$ ratio. There was no significant association between lycopene, and lutein/zeaxanthin with $\mathrm{FEV}_{1} / \mathrm{FVC}$ ratio. These results support other findings that suggest individuals, especially those with reduced pulmonary function, should increase their consumption of fruits and vegetables, especially those rich in $\alpha$-carotene, $\beta$-carotene, and $\beta$ cryptoxanthin. Further controlled dietary intervention studies are needed to determine whether an increased intake of fruits and vegetables can reverse the decrease in $\mathrm{FEV}_{1} / \mathrm{FVC}$ ratio in the adult population. 


\section{References}

1. Singh D, Agusti A, Anzueto A, Barnes PJ, Bourbeau J, Celli BR, et al. Global Strategy for the Diagnosis, Management, and Prevention of Chronic Obstructive Pulmonary Disease (2019 report). Eur Respir J [Internet]. 2019;53. Available from: https://erj.ersjournals.com/content/53/5/1900164.long

2. Vitenberga Z, Pilmane M, Babjoniševa A. The Evaluation of Inflammatory, AntiInflammatory and Regulatory Factors Contributing to the Pathogenesis of COPD in Airways. Pathol Res Pract. 2019;215(1):97-105.

3. Trends in COPD (Chronic Bronchitis and Emphysema): Morbidity and Mortality. Am Lung Assoc Epidemiol Stat Unit Res Heal Educ Div. 2013;(March):23.

4. Repine JE, Bast A, Lankhorst I. Oxidative Stress in Chronic Obstructive. Am J Crit Care Med. 1997;156(10):341-57.

5. Diagnosis of COPD [Internet]. World Health Organization. [cited 2002 Sep 20]. Available from: https://www.who.int/respiratory/copd/diagnosis/en/

6. Schünemann HJ, Freudenheim JL, Grant BJB. Epidemiologic Evidence Linking Antioxidant Vitamins to Pulmonary Function and Airway Obstruction. Epidemiol Rev. 2001;23(2):248-67.

7. Root AMM, Houser SM, Anderson JJB, Dawson HR. Healthy Eating Index 2005 and Selected Macronutrients are Correlated with Improved Lung Function in Humans. Nutr Res. 2005;(34).

8. Semba R, Chang S, Sun K, Talegawkar S, Ferrucci L, Fried L. Serum Carotenoids and Pulmonary Function in Older Community-Dwelling Women. J Nutr Heal Aging. 2012;16(4):291-6. 
9. Bousquet J, Dahl R, Khaltaev N. World Health Report. Global Alliance Against Chronic Respiratory Diseases. Allergy [Internet]. 2007;(29):233-9. Available from: http://onlinelibrary.wiley.com/doi/10.1111/j.1398-9995.2007.01307.x/full

10. Scanlon PD, Connett JE, Waller LA, Altose MD, Bailey WC, Buist SA, et al. Smoking Cessation and Lung Function in Mild-to- Moderate Chronic Obstructive Pulmonary Disease: The Lung Health Study. Am J Respir Crit Care Med. 2000;161:381-90.

11. Terzikhan N, Verhamme KMC, Hofman A, Stricker BH, Brusselle GG, Lahousse L. Prevalence and Incidence of COPD in Smokers and Non-Smokers: the Rotterdam Study. Eur J Epidemiol. 2016;31(8):785-92.

12. Barberà JA. Mechanisms of Development of Chronic Obstructive Pulmonary DiseaseAssociated Pulmonary Hypertension. Pulm Circ. 2014;3(1):160-4.

13. Lundbäck B, Lindberg A, Lindström M, Rönmark E, Jonsson AC, Jönsson E, et al. Not 15 But 50\% of Smokers Develop COPD? - Report from the Obstructive Lung Disease in Northern Sweden studies. Respir Med. 2003;97(2):115-22.

14. Campos KKD, de Oliveira Ramos C, Martins TL, Costa G de P, Talvani A, Garcia CCM, et al. Lycopene Mitigates Pulmonary Emphysema Induced by Cigarette Smoke in a Murine Model. J Nutr Biochem [Internet]. 2019;65:93-100. Available from: https://doi.org/10.1016/j.jnutbio.2018.12.008

15. Kentson M, Leanderson P, Jacobson P, Persson HL. Oxidant Status, Iron Homeostasis, and Carotenoid Levels of COPD Patients with Advanced Disease and LTOT. Eur Clin Respir J [Internet]. 2018;5(1):1447221. Available from:

https://doi.org/10.1080/20018525.2018.1447221 
16. MacNee W. Oxidants / Antioxidants and COPD. Chest. 2000;5(May):303-17.

17. Ciencewicki J, Trivedi S, Kleeberger SR. Oxidants and the Pathogenesis of Lung Diseases. J Allergy Clin Immunol. 2009;122(3):456-70.

18. Laguerre M, Lecomte J, Villeneuve P. Progress in Lipid Research Evaluation of the ability of antioxidants to counteract lipid oxidation : Existing methods, new trends and challenges. Prog Lipid Res. 2007;46:244-82.

19. Pham-Huy LA, He H, Pham-Huy C. Free Radicals, Antioxidants in Disease and Health. Int J Biomed Sci. 2008;4(2):89-96.

20. Balasaheb S, Pal D. Free Radicals, Natural Antioxidants, and Their Reaction Mechanisms. R Soc Chem. 2015;5:27986-8006.

21. Willett WC, Sacks FM, Trichopoulou A, Drescher G, Ferro-Luzzi A, Helsing E, et al. Mediterranean Diet Pyramid: A Cultural Model for Healthy Eating. Am J Clin Nutr. $1995 ; 61(6): 1402-6$.

22. Arvaniti F, Priftis KN, Papadimitriou A, Papadopoulos M, Kapsokefalou M, Anthracopoulos MB, et al. Adherence to the Mediterranean Type of Diet is Associated with Lower Prevalence of Asthma Symptoms, Among 10 - 12 Years Old Children: the PANACEA Study. Pediatr Allergy Immunol. 2011;22(6):283-9.

23. Carey O, Cookson J, Britton J, Tattersfield A. The Effect of Lifestyle on Wheeze, Atopy, and Bronchial Hyperreactivity in Asian and White Children. Am J Respir Crit Care Med. 1996;154(2).

24. Varraso R, Kauffmann F, Leynaert B, Le Moual N, Boutron-Ruault M, ClavelChapelon F, et al. Dietary Patterns and Asthma in the E3N Study. Eur Respir J. 2009;33(1):33-41. 
25. Wood LG, Garg ML, Gibson PG. A High-Fat Challenge Increases Airway Inflammation and Impairs Bronchodilator Recovery in Asthma. J Allergy Clin Immunol. 2011;127(5):1133-40.

26. Berthon BS, Wood LG. Nutrition and Respiratory Health—Feature Review. Nutrients. $2015 ; 1618-43$.

27. Butland B, Fehily A, Elwood P. Diet, Lung Function, and Lung Function Decline in a Cohort of 2512 Middle Aged Men. Thorax. 2000;55(2):102-8.

28. Wood LG, Garg ML, Smart J, Scott H, Barker D, Gibson P. Manipulating Antioxidant Intake in Asthma: A Randomized Controlled Trial. Am J Clin Nutr. 2012;96(3):53443.

29. Keranis E, Makris D, Rodopoulou P, Martinou H, Papamakarios G, Danill Z, et al. Impact of Dietary Shift to Higher-Antioxidant Foods in COPD: a Randomised Trial. Eur Respir J. 2010;36(4):774-80.

30. Meteran H, Francis S, Miller MR, Hjelmborg J, Sigsgaard T, Backer V. Self-Reported Intake of Fruit and Vegetables and Risk of Chronic Obstructive Pulmonary Disease : A Nation-Wide Twin Study. Respir Med [Internet]. 2018;144(September):16-21. Available from: https://doi.org/10.1016/j.rmed.2018.09.013

31. Palozza P, Simone RE, Catalano A, Mele MC. Tomato Lycopene and Lung Cancer Prevention: From Experimental to Human Studies. Cancers (Basel). 2011;3:2333-57.

32. Guizhou H, Cassano PA. Antioxidant Nutrients and Pulmonary Function: The Third National Health and Nutrition Examination Survey ( NHANES III ). Am J Epidemiol. 2000;151(10):975-81.

33. Grievink L, Smit HA, Ocké MC, Veer P Van, Kromhout D. Dietary Intake of 
Antioxidant (pro) -Vitamins, Respiratory Symptoms and Pulmonary Function: the MORGEN study. Thorax. 1998;166-71.

34. Kan H, Stevens J, Heiss G, Rose KM, Stephanie J, Hill C, et al. Dietary Fiber, Lung Function, and Chronic Obstructive Pulmonary Disease in the Atherosclerosis Risk in Communities (ARIC) Study. Am J Epidemiol. 2008;167(5):570-8.

35. Rao A V, Rao LG. Carotenoids and Human Health. Pharmacol Res. 2007;55:207-16.

36. Zhao LG, Zhang QL, Zheng JL, Li HL, Zhang W, Tang WG, et al. Dietary, Circulating Beta-Carotene and Risk of All-Cause Mortality: A Meta-Analysis from Prospective Studies. Sci Rep [Internet]. 2016;6(May):1-10. Available from: http://dx.doi.org/10.1038/srep26983

37. Fiedor J, Science AC. Potential Role of Carotenoids as Antioxidants in Human Health and Disease. Nutrients. 2014;466-88.

38. Eggersdorfer M, Wyss A. Carotenoids in Human Nutrition and Health. Arch Biochem Biophys [Internet]. 2018;652(May):18-26. Available from: https://doi.org/10.1016/j.abb.2018.06.001

39. Paiva SA, Russell RM. $\beta$-Carotene and Other Carotenoids. J Am Coll Nutr. 2013;18(5):325-82.

40. Stahl W, Sies H. Antioxidant Activity of Carotenoids. Mol Aspects Med. 2003;24(6):345-51.

41. Paiva SA, Godoy I, Vannucchi H, Favaro RM, Geraldo RR, Campana AO. Assessment of Vitamin A Status in Chronic Obstructive Pulmonary Disease Patients and Healthy Smokers. Am J Clin Nutr. 1996;64(6):928-34.

42. Ochs-Balcom H, Grant G, Muti P, Sempos C, Freudenheim J, Browne R, et al. 
Antioxidants, Oxidative Stress, and Pulmonary Function in Individuals Diagnosed with AsArticle, Originalthma or COPD. Eur J Clin Nutr. 2006;(November 2005):9919.

43. Grievink L, Waart FG De, Schouten EG, Kok FJ. Serum Carotenoids, $\alpha$-Tocopherol, and Lung Function among Dutch Elderly. Am J Respir Crit Med. 2000;161:790-5.

44. Willett WC, Feskanich D, Colditz GA, Speizer FE, Michaud DS, Giovannucci E, et al. Intake of Specific Carotenoids and Risk of Lung Cancer in 2 Prospective US Cohorts. Am J Clin Nutr. 2018;72(4):990-7.

45. Kirkil G, Muz MH, Sancaktar E, Kaman D, Sahin K, Kucuk O. The Effect of Lycopene Supplementation on Chronic Obstructive Lung Disease. Nobel Med. 2012;8(3):98-104.

46. Hanson C, Lyden E, Weissenburger-Moser L, Furtado J, Romberger DJ, Levan TD. Serum Level of Nutritional Antioxidants are Decreased in Veteran Smokers with COPD. J Mil Veterans Health. 2017;25(2):26-34.

47. Rautalahti M, Virtamo J, Haukka J, Heinonen OP, Sundvall J, Demetrius A, et al. The Effect of Alpha-Tocopherol and Beta-Carotene Supplementation on COPD Symptoms. Am J Respir Crit Care Med. 2013;156(5):1447-52.

48. Antioxidants: In Depth [Internet]. National Center for Complementary and Integrative Health. 2013 [cited 2019 Feb 20]. Available from:

https://nccih.nih.gov/health/antioxidants/introduction.htm

49. Joshi P, Kim WJ, Sang-Ah L. The Effect of Dietary Antioxidant on the COPD Risk: The Community-Based KoGES (Ansan-Anseong) Cohort. Int J Chron Obstruct Pulmon Dis. 2015;10:2159-68. 
50. Franklin E, Hill C. Atherosclerosis Risk in Communities Study Protocol: Cohort Component Procedures. ARIC Protoc [Internet]. 2. Available from:

https://www.ncbi.nlm.nih.gov/projects/gap/cgi-bin/GetPdf.cgi?id=phd003121.2

51. Hill C, Gerardo D, James F, Tyroler HA, Chambless LE, Romm J, et al. The Atherosclerosis Risk in Communities (ARIC) Study: Design and Objectives. Am J Epidemiol. 1989;129(4):687-702.

52. ARIC. Manual 4 - Pulmonary Function Assessment - Visit 1. ARIC Protoc [Internet]. 1987; Available from: https://www.ncbi.nlm.nih.gov/projects/gap/cgibin/GetPdf.cgi?id=phd003124.2

53. Shahar E, Folsom A, Melnick S, Tockman M, Comstock G, Shimakawa T, et al. Does Dietary Vitamin A Protect Against Airway Obstruction? ATS Journals. 1994;150(4).

54. Liu C, Bronson RT, Russell RM, Wang X. b -Cryptoxanthin Supplementation Prevents Cigarette Smoke-Induced Lung Inflammation, Oxidative Damage, and Squamous Metaplasia in Ferrets. Cancer Prev Res. 2011;(3):1255-67.

55. Schünemann HJ, McCann S, Grant BJB, Trevisan M, Muti P, Freudenheim JL. Lung Function in Relation to Intake of Carotenoids and Other Antioxidant Vitamins in a Population-based Study. Am J Epidemiol. 2002;155(5):463-71.

56. USDA Food Composition Databases [Internet]. USDA Research Service. Available from: https://ndb.nal.usda.gov/ndb/nutrients/index

57. Kelly Y, Sacker A, Marmot M. Nutrition and Respiratory Health in Adults: Findings from the Health Survey for Scotland. Eur Respir J. 2003;21(4):664-71. 


\section{Tables}

Table 1. Characteristics of Participants from the ARIC Cohort.

\begin{tabular}{|c|c|c|c|c|}
\hline Characteristic & Never smoker & Past smoker & $\begin{array}{l}\text { Current } \\
\text { smoker }\end{array}$ & $p$-value ${ }^{\mathrm{a}}$ \\
\hline$n$ & 5430 & 4471 & 3924 & \\
\hline Age at visit $1(\mathrm{y})$ & $54.1 \pm 5.8$ & $54.8 \pm 5.8$ & $53.8 \pm 5.6$ & $<0.001 * * *$ \\
\hline Female gender $(\%)$ & $74 \%$ & $38 \%$ & $48 \%$ & $<0.001 * * *$ \\
\hline Black ethnicity (\%) & $29 \%$ & $18 \%$ & $28 \%$ & $<0.001 * * *$ \\
\hline Education, post high school (\%) & $37.0 \%$ & $39.7 \%$ & $29.3 \%$ & $<0.001 * * *$ \\
\hline Standing height $(\mathrm{cm})$ & $166 \pm 9$ & $171 \pm 9$ & $170 \pm 9$ & $<0.001 * * *$ \\
\hline BMI $\left(\mathrm{kg} / \mathrm{m}^{2}\right)$ & $28.3 \pm 5.7$ & $28.1 \pm 5.0$ & $26.5 \pm 4.9$ & $<0.001 * * *$ \\
\hline Cigarette-years of smoking & 0 & $444 \pm 434$ & $637 \pm 444$ & $<0.001 * * *$ \\
\hline Total energy (kcal/day) & $1559 \pm 567$ & $1625 \pm 606$ & $1662 \pm 634$ & $<0.001 * * *$ \\
\hline Total cholesterol $(\mathrm{mmol} / \mathrm{L})$ & $5.5 \pm 1.1$ & $5.6 \pm 1.1$ & $5.6 \pm 1.1$ & 0.126 \\
\hline Total carotenoids (mg/day) & $9.2 \pm 6.4$ & $8.9 \pm 6.5$ & $8.6 \pm 6.5$ & $<0.001 * * *$ \\
\hline Total physical activity score & $6.9 \pm 1.4$ & $7.1 \pm 1.4$ & $6.8 \pm 1.5$ & $<0.001 * * *$ \\
\hline $\mathrm{FEV}_{1} / \mathrm{FVC}$ for visit $1(\%)$ & $77 \pm 6$ & $74 \pm 7$ & $71 \pm 9$ & $<0.001 * * *$ \\
\hline
\end{tabular}

a. $p$ values are for the comparison between never smokers, past smokers, and current smokers.

${ }^{*} p<0.05,{ }^{* *} p<0.01,{ }^{* * *} p<0.001$ 
Table 2. Association Between Carotenoid Intake and $\mathrm{FEV}_{1} / \mathrm{FVC}$ Ratio.

\begin{tabular}{|c|c|c|c|}
\hline & Beta $^{a}$ & Confidence Interval & p-value \\
\hline Univariate & 0.024 & $0.004,0.043$ & $0.016 *$ \\
\hline Demographic $^{\mathrm{b}}$ & 0.018 & $-0.001,0.037$ & 0.057 \\
\hline Multivariate $^{c}$ & 0.020 & $0.002,0.039$ & $0.033 *$ \\
\hline
\end{tabular}

a. Beta value is the change in FEV1/FVC ratio for each $\mathrm{mg}$ increase in total carotenoid intake.

Units of beta $\mathrm{FEV}_{1} / \mathrm{FVC} \%$ per mg carotenoid

b. Demographic linear regression controls for sex, age, and race

c. Multivariate linear regression controls for sex, age, education level, BMI, total calorie intake, standing height, smoking status, cigarette-years of smoking, physical activity level, and total serum cholesterol level.

${ }^{*} p<0.05, * * p<0.01, * * * p<0.001$ 
Table 3. Association Between Each Carotenoid Intake and $\mathrm{FEV}_{1} / \mathrm{FVC}$ Ratio.

\begin{tabular}{llll}
\hline & Beta & Confidence Interval & p-value \\
\hline$\alpha$-carotene & 0.338 & $0.159,0.517$ & $<0.001^{* * *}$ \\
$\beta$-carotene & 0.080 & $0.027,0.133$ & $0.003^{* *}$ \\
$\beta$-cryptoxanthin & 1.868 & $0.506,3.229$ & $0.007^{* *}$ \\
Lycopene & 0.018 & $-0.015,0.050$ & 0.283 \\
Lutein and Zeaxanthin & 0.004 & $-0.045,0.054$ & 0.861 \\
& & & \\
With Vitamin C as a Covariate & & Confidence Interval & $\boldsymbol{p}$-value \\
\hline \multicolumn{5}{l}{ Total carotenoids } & 0.017 & $-0.002,0.037$ & 0.078 \\
$\alpha$-carotene & 0.324 & $0.142,0.505$ & $<0.001^{* * *}$ \\
$\beta$-carotene & 0.074 & $0.019,0.129$ & $0.008^{* *}$ \\
$\beta$-cryptoxanthin & 1.864 & $0.190,3.539$ & $0.029^{*}$ \\
Lycopene & 0.014 & $-0.018,0.047$ & 0.394 \\
Lutein/zeaxanthin & -0.005 & $-0.056,0.046$ & 0.844
\end{tabular}

Units of beta $\mathrm{FEV}_{1} / \mathrm{FVC} \%$ per mg carotenoid

Multivariate linear regression same as Table 2.

${ }^{*} p<0.05, * * p<0.01, * * * p<0.001$ 
Table 4. Servings of Carotene-Rich vs. Lycopene and Lutein/Zeaxanthin Fruits and Vegetables Across Smoking Status.

\begin{tabular}{lllll}
\hline Characteristic & $\begin{array}{l}\text { Never } \\
\text { smoker }\end{array}$ & $\begin{array}{l}\text { Past } \\
\text { smoker }\end{array}$ & $\begin{array}{l}\text { Current } \\
\text { smoker }\end{array}$ & $p$-value \\
\hline F \& V rich in carotenes & $1.7 \pm 1.2$ & $1.6 \pm 1.1$ & $1.3 \pm 1.1$ & $<0.001 * * *$ \\
\hline F \& V rich in lutein/zeaxanthin & $1.1 \pm 0.7$ & $1.0 \pm 0.7$ & $1.0 \pm 0.7$ & 0.343 \\
\hline \multicolumn{2}{l}{ Values are expressed as percentages or as mean $\pm S D$, P value is based on } \\
ANOVA statistics
\end{tabular}


Table 5. Association Between Carotenoid-Rich Food Sources and FEV $1 / \mathrm{FVC}_{\text {Ratio. }}$

\begin{tabular}{llll}
\hline & Beta & Confidence Interval & $p$-value \\
\hline F \& V rich in carotenes & 0.146 & $0.038,0.253$ & $0.008 * *$ \\
F \& V rich in lycopene \& lutein/zeaxanthin & 0.061 & $-0.124,0.245$ & 0.518 \\
Units of beta $\mathrm{FEV}_{1} / \mathrm{FVC}_{0}$ per serving of carotenoid-containing foods. & \\
Multivariate linear regression same as Table 2. & & \\
$\quad * p<0.05, * *_{p}<0.01, * * * p<0.001$ &
\end{tabular}




\section{Vita}

Lauren Yourim Jun was born in South Korea to Jaeeun and Jongil Jun. She graduated from Eugene Ashley High school in Wilmington, NC, in 2013. She earned a Bachelor of Science degree in Nutrition and Dietetics from Appalachian State University in May 2018. She continues her education in dietetics at Appalachian State University through the combined Master of Science in Nutrition and Dietetic Internship program beginning in 2018. Ms. Jun resides in Oxford, AL. 